\title{
Multidetector CT in Renal Tuberculosis
}

\author{
Ashwini Sankhe $\cdot$ Anagha R. Joshi
}

Published online: 12 September 2014

(C) Springer Science+Business Media New York 2014

\begin{abstract}
Tuberculosis (TB) is one of the commonest infectious diseases in the developing countries with rising incidence seen due to HIV and AIDS. Pulmonary tuberculosis is the commonest form seen. Renal Tuberculosis is the most common extrapulmonary form. There are no specific clinical symptoms associated with renal tuberculosis. The constitutional symptoms like low grade fever, weight loss, anorexia commonly seen with pulmonary TB are not usually seen with renal TB. Renal TB is also cause of interstitial nephritis which leads to end-stage renal failure. Hence the role of radiologist assumes importance in supporting the timely diagnosis although cultures or histologic analysis is required for definitive diagnosis. The individual imaging finding may not be diagnostic as it can be seen with other renal pathologies too, however, presence of multiple imaging findings in a single case is pointer to this disease. Renal TB involves both cortex and collecting system. This article gives review of pathophysiology and computed tomography features of Renal TB.
\end{abstract}

Keywords Extrapulmonary tuberculosis - CT urography · Renal abscess $\cdot$ Papillary necrosis $\cdot$ Caliectasis $\cdot$ Putty kidney

This article is part of Topical Collection on Abdominal CT Imaging.

A. Sankhe $(\bowtie)$. A. R. Joshi

Lokmanya Tilak Municipal Medical College and General Hosp.

Sion, Mumbai 400022, Maharashtra, India

e-mail:drashraj@yahoo.com

A. R. Joshi

e-mail: anagha.j@rediffmail.com

\section{Introduction}

Renal tuberculosis is a subset of genitourinary tuberculosis accounting for $20 \%$ of extrapulmonary tuberculosis. Genitourinary tuberculosis is the most common form of extrapulmonary tuberculosis [1]. Globally, tuberculosis is a common disease, with 8-10 million new cases annually and a rising incidence, particularly in regions with a high incidence of HIV infection [2]. Approximately $95 \%$ of cases occur in developing countries. In India, more than 1,000 lives are lost every day due to TB despite the availability of modern diagnostic aids and treatment [3].

Tuberculosis can involve both the renal parenchyma and the collecting system (calyces, renal pelvis, ureter, bladder, and urethra) and results in different clinical presentations and radiographic appearances. This article focuses on the role of computed tomography in evaluation of renal parenchyma, calyces, and renal pelvis. The appearance of renal TB-CT is shown in Flowchart 1.

Pathogenesis

Renal and urinary tract tuberculosis is generally caused by the human tubercle bacillus, M. tuberculosis, but the bovine tubercle bacillus, M. bovis, can be responsible occasionally. The intravesical instillation of Bacille Calmette-Guérin vaccine done for the treatment of superficial bladder cancer can also result in renal lesions as a complication [2].

Renal Tuberculosis occurs through hematogenous spread from a primary focus like lung and is usually a bilateral disease [4]. The pathogenesis of this disease begins with the initial localization of the tubercle bacilli in the cortex adjacent to the glomeruli causing mechanical stress which leads to alteration in the cell morphology, 


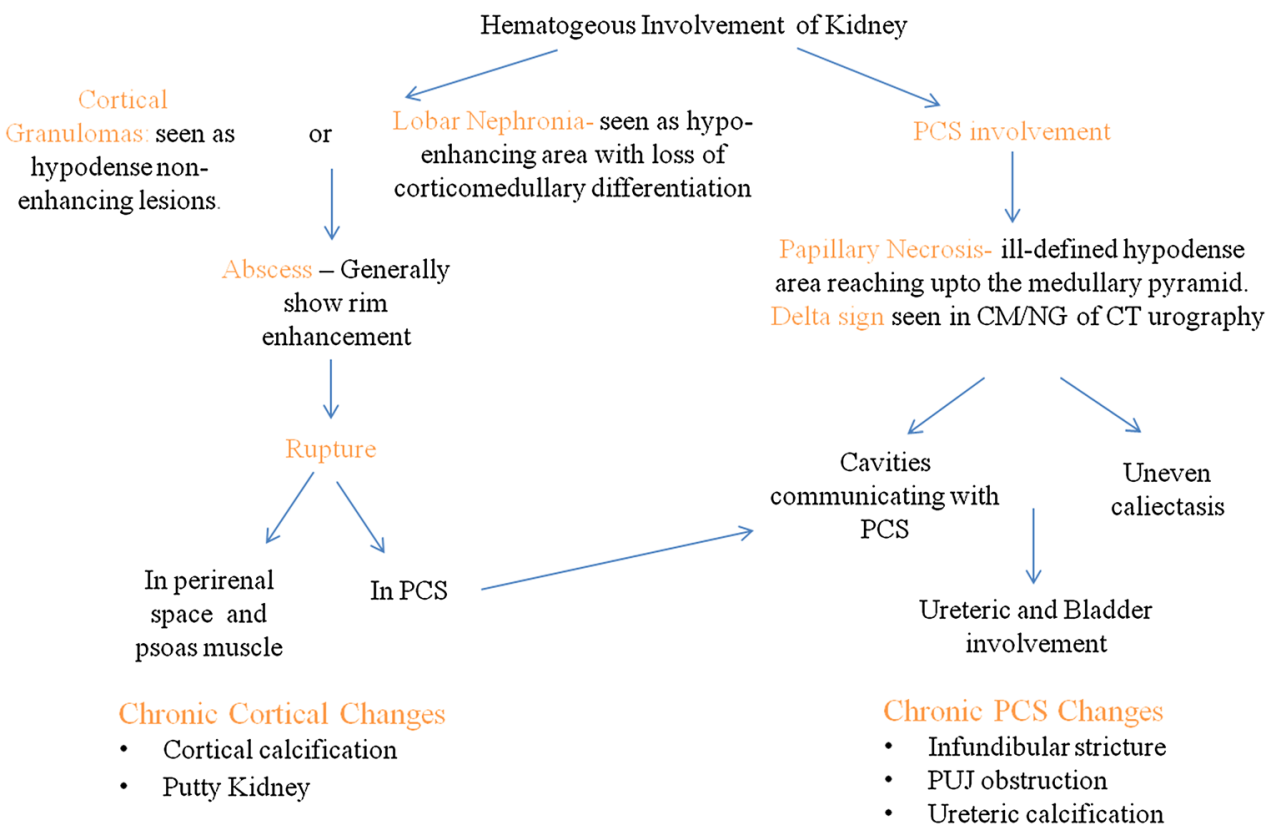

Multiplicity and Lobar caseation is the hallmark of TB

Flowchart 1 Renal TB-CT appearance

increased rate of protein synthesis and proliferation of resident glomerular cell [4]. The cortical granulomas may remain dormant, asymptomatic, and stable as sequelae of a primary pulmonary infection from as long ago as 10-15 years (Khan et al. 2004). Renal tuberculosis may occur as a result of reactivation after this period of dormancy, even when there is no evidence of active pulmonary tuberculosis, or it may be due to reinfection from active tuberculosis (Ahmed and Murty 2003) [5]. If the infection is not controlled in this phase, the organism then gains access to the tubular fluid by the sloughing of small glomerular caseating necrotic lesions. While within tubules, further dissemination of the bacilli to the renal medulla and pyramidal tissues can take place. This leads to papillitis that extends into the proximal loop of Henle and eventually papillary necrosis occurs. Thereafter, the degree of progression varies from self-limiting process to advanced destructive lesions of the kidneys. The infection may remain localized in the renal parenchyma and/or gain access to the calyceal system causing pyelocalyceal destruction and subsequently the ureters and urinary bladder become involved. This may progress to hydronephrosis and pyonephrosis secondary to strictures and obstruction [4]. As in the primary site, granuloma formation, caseous necrosis, and cavitation within the renal parenchyma are the hallmark stages of progressive infection [5].

The host's response induces fibrotic parenchyma which may lead to renal pelvic traction, calcium deposition with stone formation within the urinary collection system, and stricture formation contributing to obstruction and progressive renal dysfunction. The end-stage result of diffuse disease is destruction, loss of function, fibrosis, and calcification in a lobular distribution of the entire kidney producing autonephrectomy [18]. If granulomas spread to the collection system, regional spread by either ascension or descension of the bacilli to the renal pelvis, ureters, urinary bladder, and accessory genital organs is possible $[4,5]$.

\section{Clinical Features}

There are no early symptoms in renal TB. Presenting symptoms can be hematuria, nocturia, dysuria, pyuria, increased frequency, back pain, suprapubic pain. These symptoms are also seen with bacterial cystitis and in most cases renal TB is suspected only after usual antibacterial treatment is ineffective [2]. Constitutional symptoms like malaise, anorexia, low grade fever, weight loss commonly seen with pulmonary TB are generally not seen with renal TB.

\section{Laboratory Diagnosis}

Tuberculosis can be diagnosed histopathologically by biopsy material on routine solid LJ media, microbiologically by isolation of mycobacterium from urine or by radiometry. Urine samples of first void are preferred over 
24-h urine collections as mycobacterial viability decreases with prolonged exposure to acid urine $[5,6]$.

Newer rapid detection techniques based on nucleic acid amplification technique like polymerase chain reaction (PCR) are being used recently for diagnosing mycobacterium tuberculosis and other environmental mycobacteria. PCR is especially efficient in detecting mycobacterium from sputum sample. PCR technique is also specific and sensitive for diagnosing genitourinary TB [2, 7]. PCR is also used in urine samples of disseminated tuberculosis related to $\mathrm{HIV}$ and identifies mycobacterial DNA where traditional methods are not specific and sensitive [8]. GeneXpert MTB/RIF is relatively new technique used for detection of mycobacterium by identifying DNA sequence specific for $\mathrm{TB}$ and rifampicin resistance by PCR.

\section{Computed Tomography}

Diagnosis of renal tuberculosis may be delayed or missed as the disease may not cause symptoms in early stages or maybe clinically misdiagnosed for other renal diseases causing similar symptoms. Hence the radiological investigations play an important role in reaching the diagnosis. Earlier Intravenous pyelography assumed an important role in diagnosing the extent of disease however the current generation multidetector CT scanner with short scanning time and a minimum slice thickness, achieve high diagnostic accuracy with good resolution, excellent sagittal and coronal reformation, and minimum artifacts.

CT is helpful in determining the extrarenal spread of tuberculous disease as well as in assessing its severity in terms of loss of renal function [9, 10•]. It is the most sensitive modality for identifying renal calcifications which occur in almost $50 \%$ cases of renal TB [9]. CT is also the best modality for demonstrating the extent, nature, and distribution of calcification within the abnormal kidney [10•]. CT, in general, shows more details of pathologic anatomy due to the availability of axial images for review and is superior to retrograde pyelography (RGP), IVU, and USG in detecting multiple small urothelial lesions [10•]. CT urography is generally performed for evaluation of renal pathologies.

We perform CT Urography with $75 \mathrm{ml}$ of $300 \mathrm{mg} \%$ of non-ionic contrast medium followed by saline injection. Generally four phases are taken which includes unenhanced scan followed by corticomedullary phase, nephrographic, and pyelographic i.e., excretory phase. Unenhanced CT is performed from upper pole of kidneys upto the symphysis pubis. It is used to evaluate calcification, fat, and baseline-unenhanced Hounsfield values of masses so that comparable enhancement of lesions can be done in contrast-enhanced scans. Corticomedullary phase is not employed by some groups as arterial phase information is generally not required [11•].

CT features of renal TB are varied and depend upon the stage of the disease. They result from a combination of papillary necrosis and parenchymal destruction. Typically, the papillae are involved first and this is followed by cortical damage. Communication with the collecting system results in thickening, ulceration, and fibrosis, often with stricture formation [10•, 12, 13] and consequent obstruction. The most valuable feature of renal TB is the multiplicity of abnormal findings [5, 12-14]. A lobar pattern of caseation, arising from the assimilation of the calyces into the caseous parenchyma of each destroyed lobe, is virtually diagnostic of renal TB.

Imaging characteristics can be divided into renal parenchymal changes and pelvicalyceal system changes. Renal Tuberculosis can also be classified according to manifestation as extensive cavitation (open or extensive forms) or fibrosclerosis (closed forms) [11•]

\section{Renal Parenchymal Changes}

Early changes in renal TB include granulomas of $\leq 3 \mathrm{~mm}$ in size and papillary necrosis. Both corticomedullary and nephrographic phases need to be carefully evaluated for these. A TB granuloma is seen as a solid mass with little or minimal enhancement after contrast administration [14] (Fig. 1) and is usually accompanied by collecting system changes [14]. In rare cases, there may be single or multiple parenchymal nodules, without collecting system involvement [15]. The nodules are variable-sized, well-defined parenchymal lesions on cross-sectional images and may mimic renal neoplasms, which may lead to unnecessary

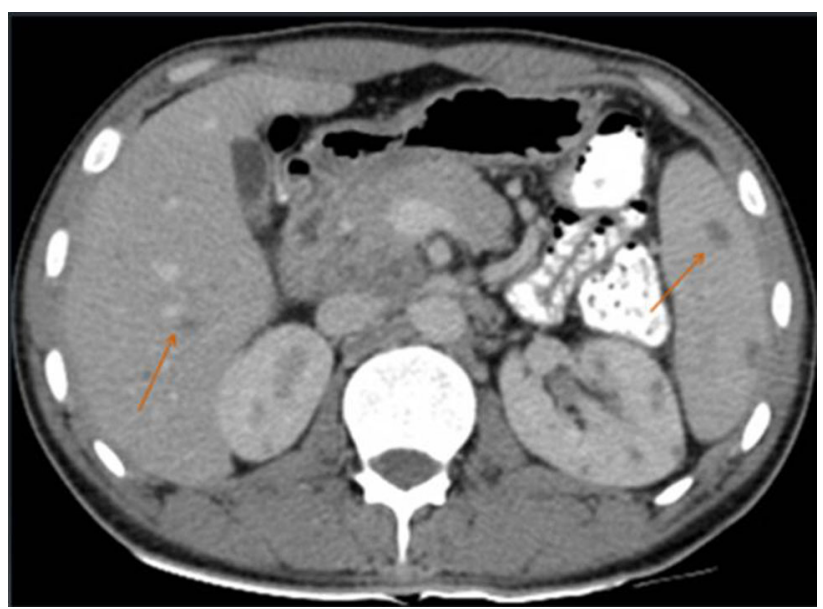

Fig. 1 Multiple hypodense lesions in both kidneys, spleen and liver s/o granulomas 


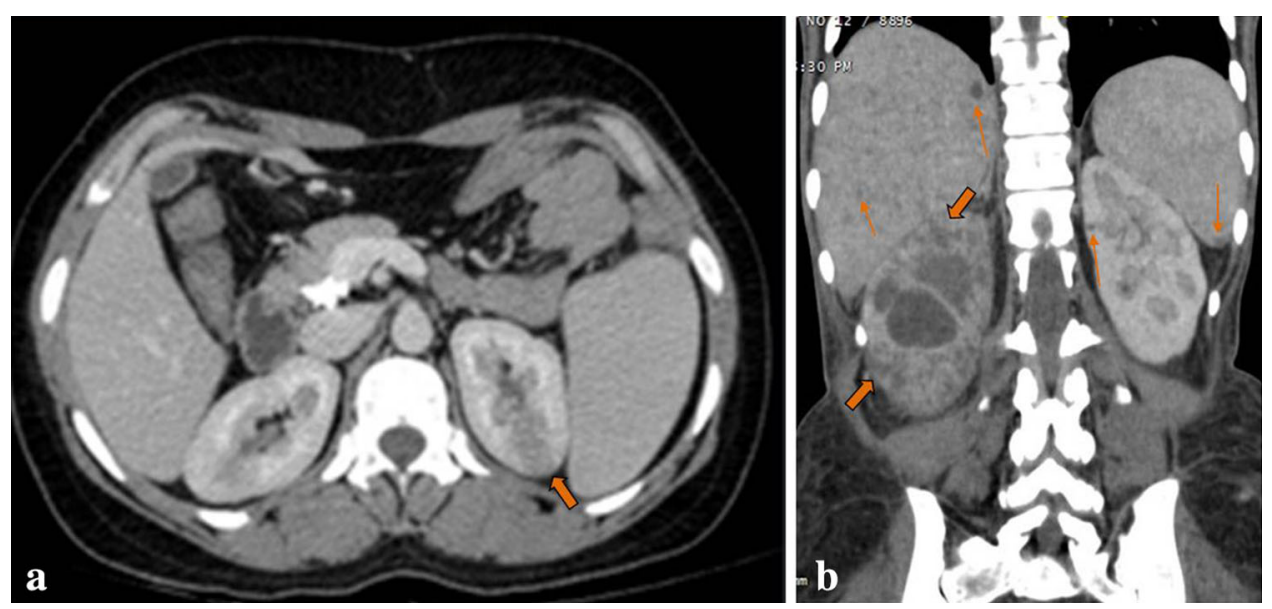

Fig. 2 a Nephrographic phase reveals hypodensity with loss of cortico medullary differentiation (arrow) involving the left kidney mimicking lobar nephronia, b coronal MPR reveals multiple hypodense lesions s/o granulomas seen in liver, spleen, both kidneys shown by thin arrows. Also note areas of focal hypoperfusion in right kidney represented by thick arrows
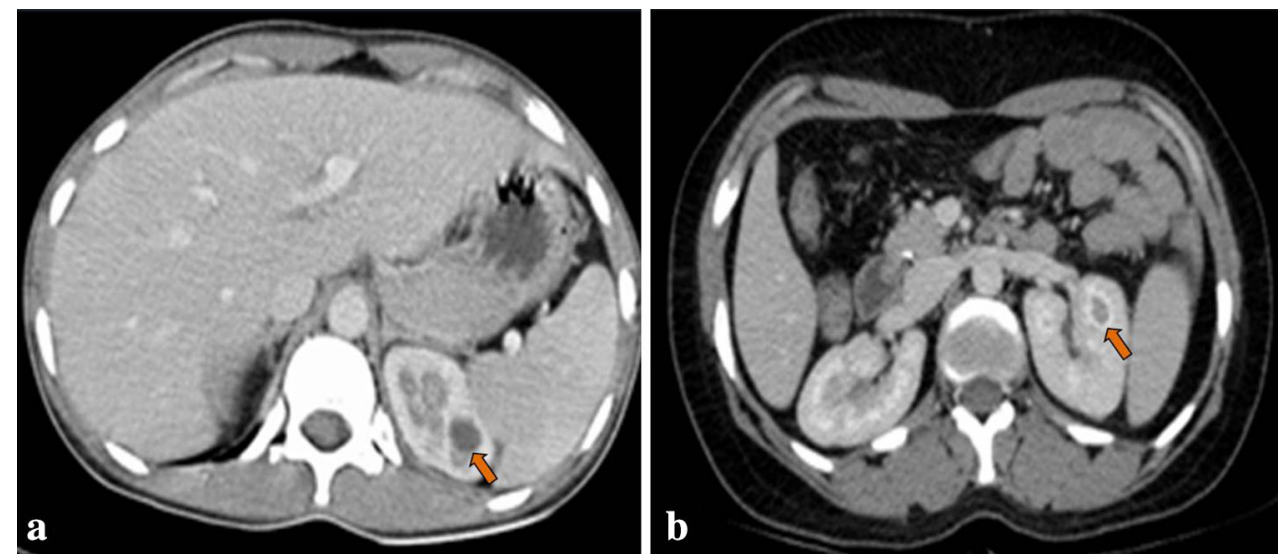

Fig. 3 a Non-enhancing abscess in left kidney, b peripheral enhancing hypodense lesion in left kidney s/o abscess

surgery; these are therefore labeled as the 'pseudo-tumoral' type [15-17].

Localized tissue edema and vasoconstriction caused by active inflammation results in focal hypoperfusion as seen on nephrographic phase of CT Urography, a finding similar to that seen in acute pyelonephritis caused by other organisms [18] (Fig. 2a, b). Coalesced cortical granulomas containing either caseous or calcified material are readily identified at CT [9]. The majority reveals evidence of peripheral enhancement [19]. Renal abscesses thus formed, can grow to large sizes and may show air foci within specially if secondary infection sets in. These abscesses may rupture and spread into the perirenal space and beyond. Tubercular renal abscesses are seen as hypodense areas of 10-40 HU with mild peripheral enhancement (Fig. 3a, b). Cavitation within the renal parenchyma may be seen as irregular pools of contrast material if a calyceal communication exists [20]. Focal, segmental, and polar involvement can be demonstrated. Inflammatory granulomatous and caseating masses show enhancement [21].

In a series of 50 patients by Lu et al. [22] the commonest finding (68\%) was of one or more cavities adjacent to a calyx, with thinning of the adjacent cortex (Fig. 4). Cortical thinning is a common CT finding and may be either focal or global (Figs. 4, 5). Parenchymal scarring is readily apparent at CT [9]. Focal or diffuse cortical scarring and non-function are other findings of advanced disease (Fig. 6) [23]. Calcifications within the renal parenchyma are common (occurring in $37-71 \%$ of cases; Fig. 6) and follow a variety of patterns. Calcifications may be amorphous, granular, lobar or curvilinear, and frequently extend beyond the kidney (e.g., psoas muscle) [11•].

Renal calcifications have also been described in atypical mycobacterium infections, viz, Mycobacterium avium-intracellulare [24], which have been reported in $5.5 \%$ of patients with AIDS. These usually take the form of fine 

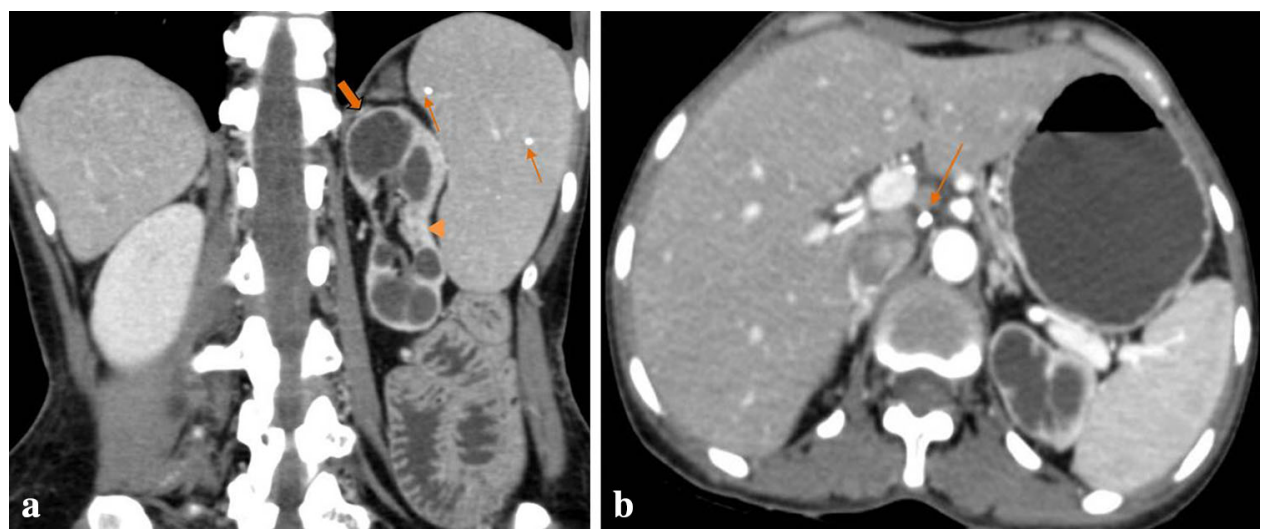

Fig. 4 a, b Coronal MPR and axial nephrographic phase images reveal caliectasis with cortical thinning (thick arrow) and parenchymal scarring (arrowhead) of left kidney. Also note splenic calcifications (thin arrow). Calcified mesenteric node (arrow) seen in axial section

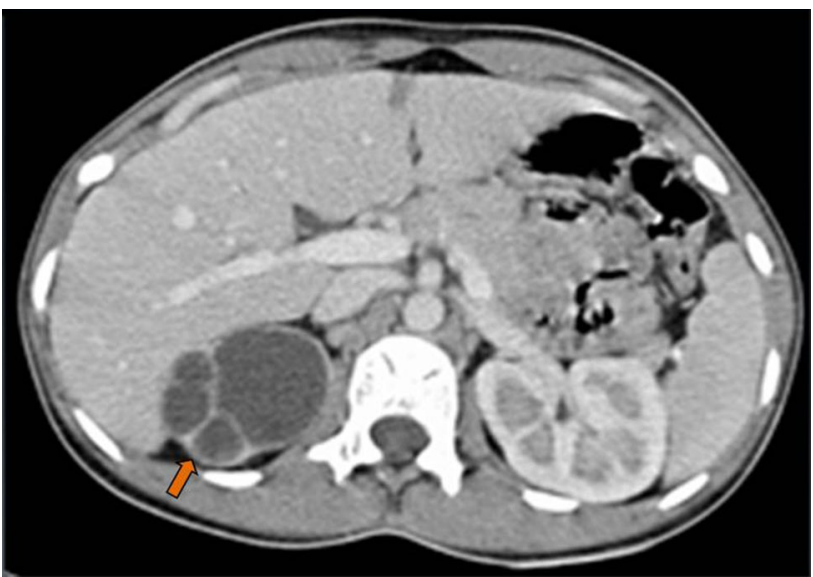

Fig. 5 Uneven calicectasis with parenchymal thinning of right kidney

punctuate calcification and as a rare exception to the usual rule in mycobacterial calcification-occur in the acute rather than the chronic stage of disease. A high level of suspicion is usually necessary and biopsy specimens must be routinely stained for acid-fast bacilli and cultured for $M$. avium-intracellulare [10•].

\section{Pelvicalyceal System (PCS) Involvement}

These cortical granulomas can colasece, cavitate forming renal abscess as described earlier or they may rupture/ communicate with the collecting system leading to spread of tuberculous bacilli into the renal pelvis, ureters, urinary bladder, and accessory genital organs. There are three possible pathways for evolution of the disease: extensive cavitation, fibrosclerosis with resulting non-communicating cavities, and recurrent 'pousséesis [11•] (meaningexacerbation). The fibrosing reaction results in stricture formation, obstructive hydronephrosis while extensive cavitation causes renal caseation.

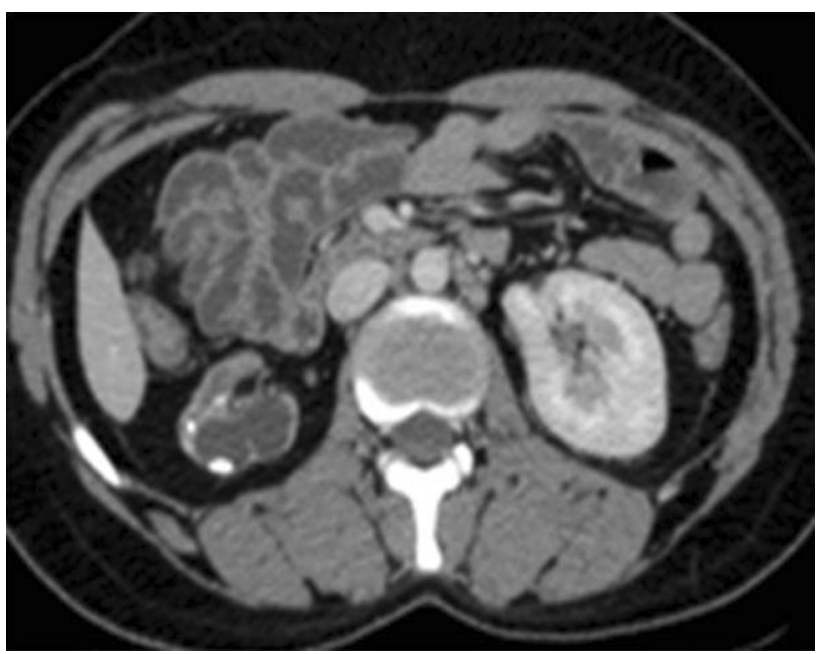

Fig. 6 Atrophic, scarred right kidney with hydronephrosis seen. Also noted are parenchymal calcifications

Lang [25] studied 86 patients with microscopic hematuria and negative IVUs on multiphasic MDCT; papillary and medullary necrosis was positively identified in 25 (29\%) of these patients. Papillary necrosis is ischemic necrosis which can be focal or diffuse. In the ischemic stage, papillary necrosis is seen as an ill-defined area of decreased enhancement at the tip of medullary pyramid seen on corticomedullary as well as nephrographic phase; though best depicted on nephrographic phase (Fig. 7) [26]. The early ischemic stage findings are depicted on Multidetector CT and are not seen on Intravenous pyelography. Another advanced imaging finding is medullary cleft that originates from fornices and extend into the medullary pyramids and papillae causing sloughing of the papillae. Necrosis of papillae results in contrast-filled cavities, seen in excretory phase (Fig. 8) [26], the sloughed papillae itself may be seen as filling defect. Papillary necrosis is seen in three forms; medullary (centrally located), papillary 
(peripherally located) and necrosis in situ. Excretory phase CT thus allows the diagnosis of renal papillary necrosis, although its sensitivity for detection of the condition has not been determined $[27,28]$. In the last stage, as papillae heal and epithelialize, blunted appearance of calyces is

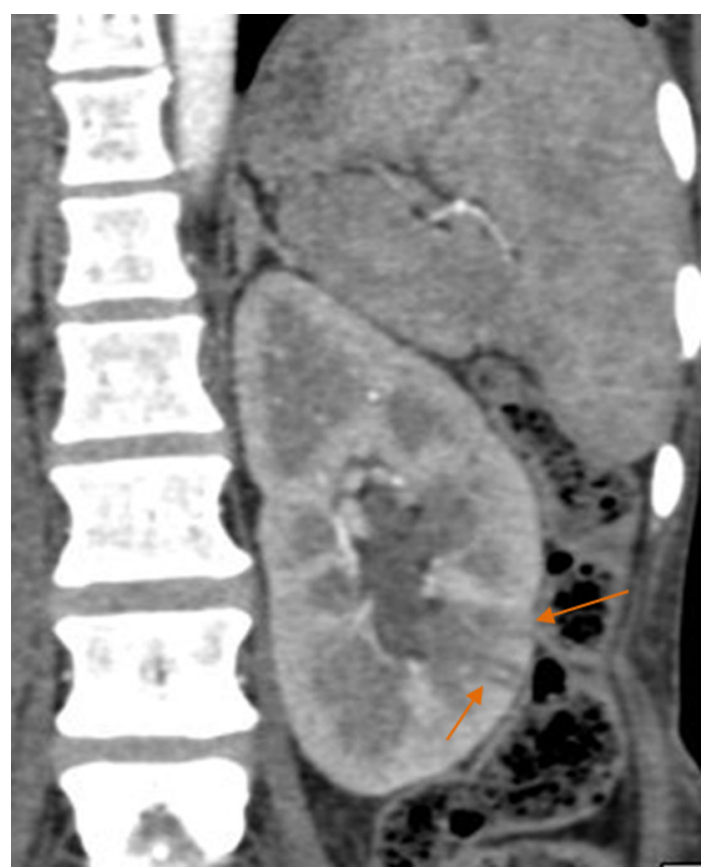

Fig. 7 Corticomedullary phase-magnified Coronal MPR reveals illdefined areas of decreased enhancement reaching up to medullary pyramid s/o necrosis seen. Also noted is renal contour changes with parenchymal atrophy and scarring.

Papillary necrosis though seen as early finding in renal $\mathrm{TB}$, it is not specific as it is seen with many other conditions like obstructive uropathy, diabetes, analgesic abuse or overuse, sickle cell disease, pyelonephritis.

The most characteristic finding seen in renal TB is uneven caliectasis (Figs. 5, 9) $[15,16,20]$ caused by varying degrees of fibrosis and obstruction at different sites [18]. Various patterns of hydronephrosis may be seen on CT depending on the site of the stricture; these include focal caliectasis, caliectasis without pelvic dilatation [12], and generalized hydronephrosis (Fig. 10). Fibrotic strictures of the infundibuli and renal pelvis may be seen on CECT [9]. Caliectasis that is not revealed by the IVU because of poor renal function, can be well assessed on CT. Calices that are dilated and filled with fluid have an attenuation between 0 and $10 \mathrm{HU}$; debris and caseation, between 10 and $30 \mathrm{HU}$; putty-like calcification, between 50 and $120 \mathrm{HU}$; and calculi, greater than $120 \mathrm{HU}$ [9]. The development of infundibular, pelvic or ureteral strictures (Fig. 10) is nearly pathognomonical of renal tuberculosis $[11 \cdot, 29]$

$\mathrm{CT}$ is not as sensitive as excretory urography in the detection of early urothelial mucosal changes [9]. The newer generation multidetector $\mathrm{CT}$ is helpful in detection of urothelial thickening because of its inherent excellent resolution (Fig. 9b, c) shows the urothelial thickening. Kenny [29] stated that although pelvi-infundibular strictures, papillary necrosis, cortical low-attenuating masses, scarring, and calcification may be seen in other conditions,
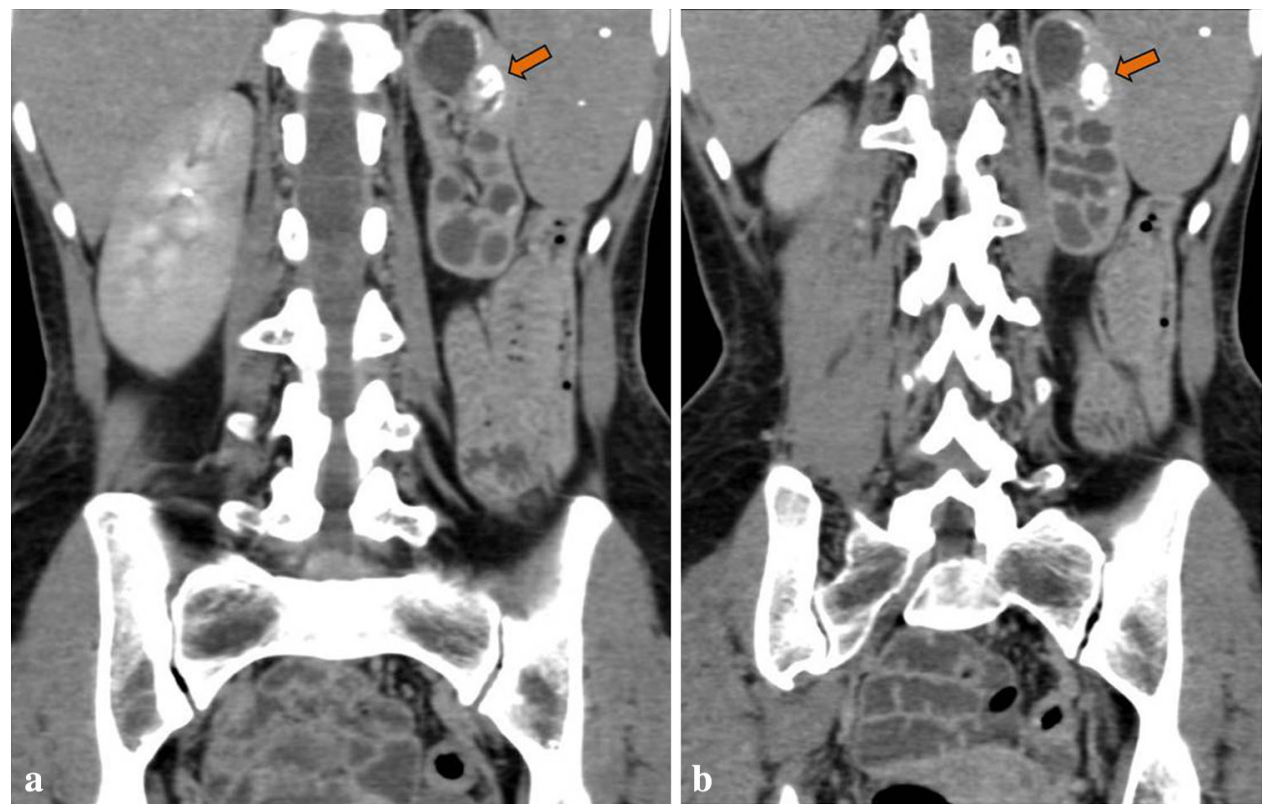

Fig. 8 a, b Excretory phase of MDCT reveals opacification of cavity with contrast. Also note splenic calcifications 

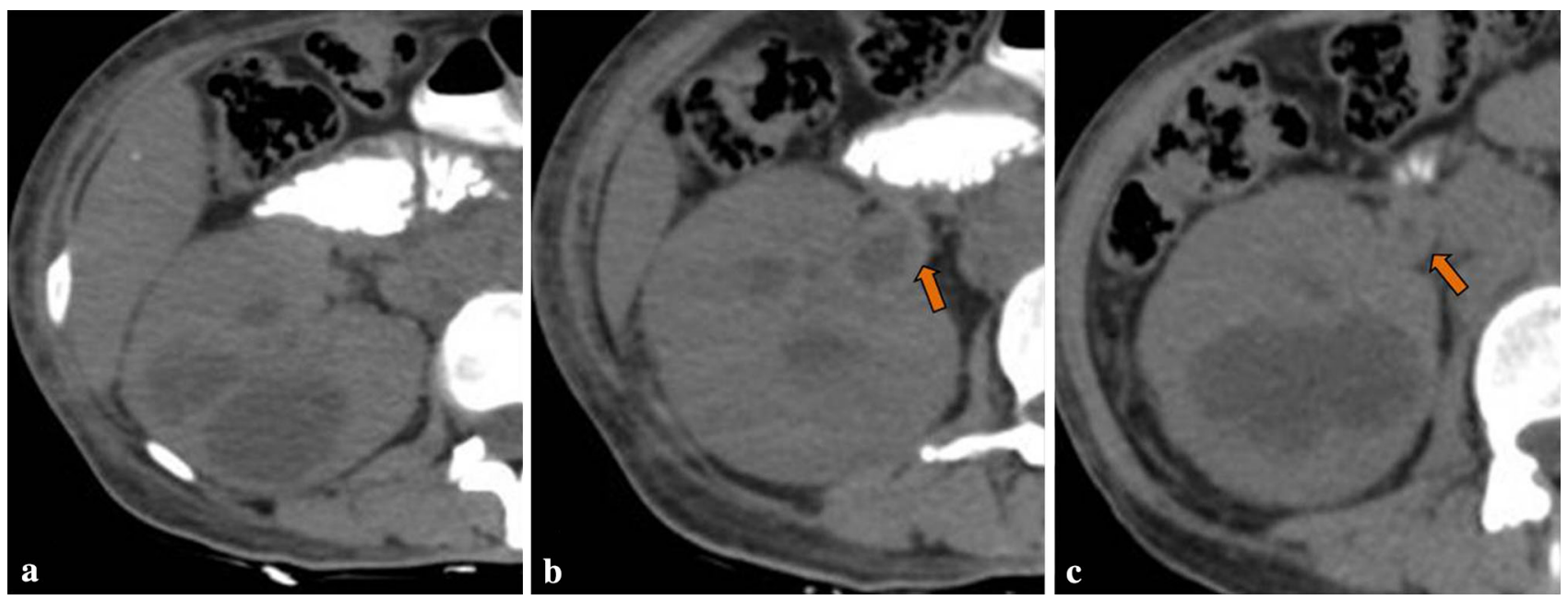

Fig. 9 a-c Plain axial CT reveals uneven caliectasis with urothelial thickening (arrows) of the pelvis causing stricture

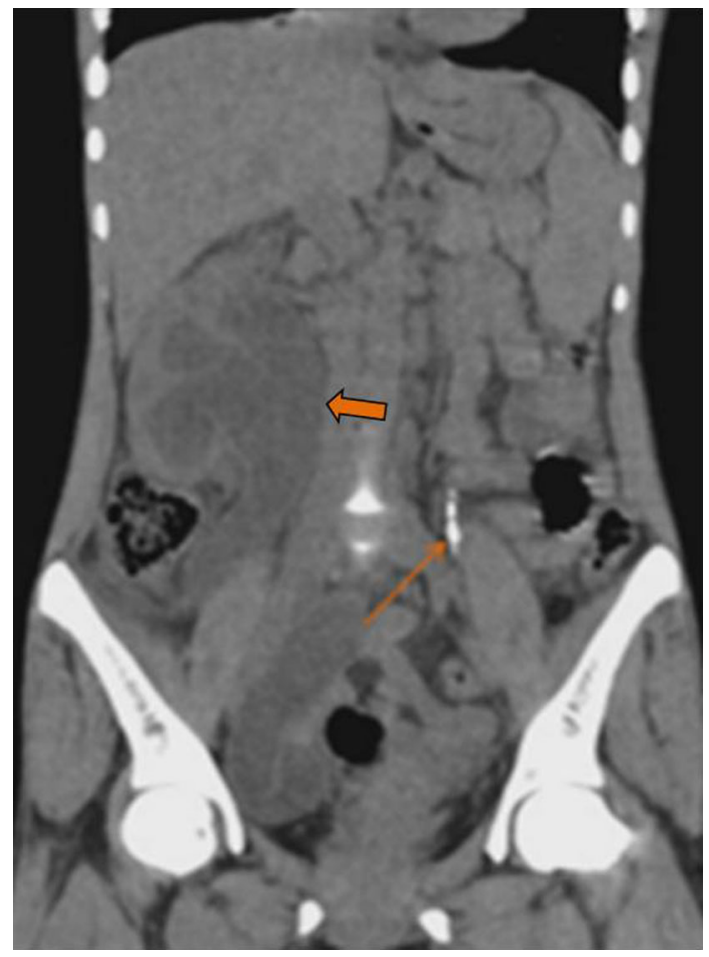

Fig. 10 Left ureteral calcification (thin arrow) with generalized hydronephrosis (thick arrow) of right kidney

the combination of three or more of these findings is highly suggestive of $\mathrm{TB}$, even in the absence of documented pulmonary disease. In one study, $35 \%$ of patients had four of these findings and $40 \%$ had three [30]. A lobar pattern of caseation, arising from the assimilation of the calyces into the caseous parenchyma of each destroyed lobe, is virtually diagnostic of renal TB (Fig. 11a, b).

As the infection and lobar caseation progresses it results in formation of putty kidney; the entire dilated collecting system is filled with calcified and thick material (Fig. 12)
[11•]. The kidney becomes small, scarred, and densely calcified [14], with autonephrectomy.

Individual $\mathrm{CT}$ features may not be pointers toward Renal TB as each renal CT feature is a 'great mimicker' [11•] of other pathologies. However multiple imaging findings like caseation, pelvicalyceal strictures, calcifications, parenchymal scarring aid in the diagnosis. CT findings of TB elsewhere in the body, for example, features of abdominal TB such as ascites (especially with septations), omental infiltration (Fig. 13), peritoneal thickening, mesenteric involvement, bowel wall thickening, enlarged lymph nodes (especially those with necrosis), splenomegaly, and splenic or hepatic focal lesions, are also pointers toward the possibility of the urinary tract lesion being of TB origin [31].

\section{Renal TB in Immunocompromised Patients}

Significant number of immunocompromised patients reveal predominantly parenchymatous renal involvement (87.5 vs. $6.2 \%$ ) [32]. In such individuals, granulomas may be less well formed and caseous necrosis seen less frequently [33]. When immune suppression is severe, and in cases in which the infective organism is one of the environmental mycobacteria, e.g., M. avium-intracellulare, the lesions may be more diffuse and poorly formed and the usual miliary lesions and caseation may not be a prominent feature [33]. A lower frequency of stenosis of the collecting system (12.5 vs. $93.8 \%$ ) and contracted bladder (12.5 vs. $65.3 \%$ ) has been noted in HIV positive patients [32]. There is disseminated involvement of tuberculosis seen in the patients with low CD4 counts especially in those less than $50 / \mathrm{cu} \mathrm{mm}$ or lower with kidney being involved incidentally with pathological condition like granulomatous interstitial nephritis [2]. 
Fig. 11 a, b Plain CT Coronal MPR reveals lobar caseation (thin arrow) with amorphouscalcified material (thick arrow) in pelvicalyceal system.

Cortical thinning, parenchymal scarring of left kidney is seen. Note there is no dilatation of pelvis seen
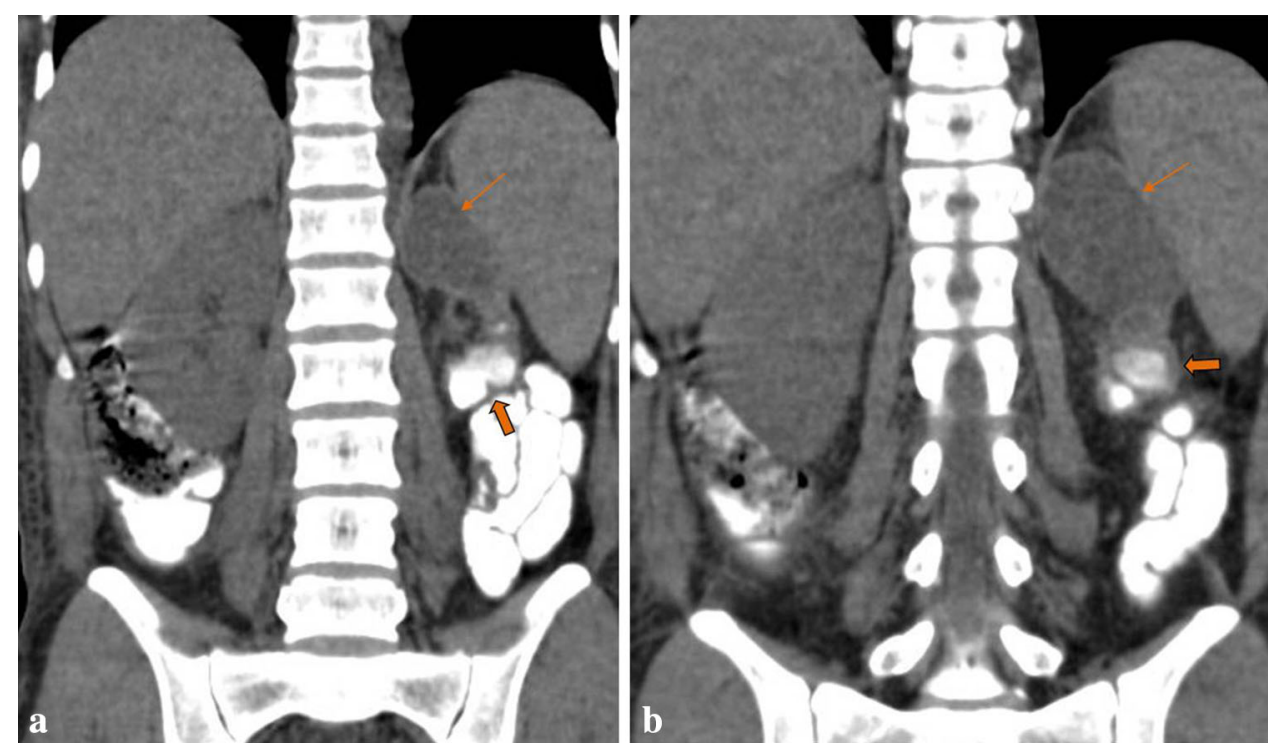

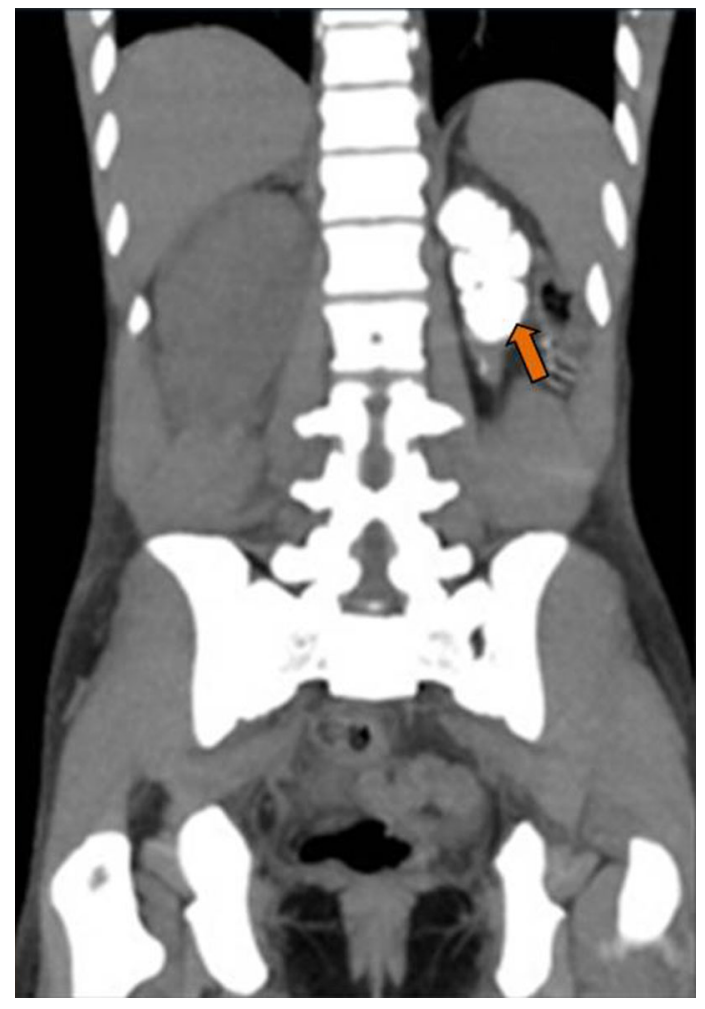

Fig. 12 Plain coronal MPR reveals left putty kidney

In an autopsy study in India [2, 34], 24 of 35 kidneys from patients who died of AIDS showed evidence of infection, including 17 cases of tuberculosis. In a similar study in Mexico City, renal disease was demonstrable in 87 of $138(63 \%)$ autopsies on AIDS patients: infection was the cause of the renal disease in 36 cases, with 19 being due to $M$. tuberculosis [2, 35].

Association between TB and Non Hodgkin lymphoma has been seen in immunodeficient individual [36].
Tuberculosis and Vitamin D Deficiency

It has been found that fall in serum 25-OH-vitamin D levels compromises cell-mediated immune defenses and leads to the activation of latent tuberculosis [2, 37]. In a predominantly vegetarian population of Asians of Gujarati origin living in West London, those with undetectable serum 25-OH-vitamin D levels had an almost tenfold increased risk of active tuberculosis [2, 38].

\section{Transplant Patients}

Tuberculosis is one of the serious complicating factor in renal and other forms of transplantation, with an incidence, depending on geographic region, of $0.35-15.0 \%$ [39]. Transplant patients have low immunity due to immunosuppressed drugs and therefore more prone for different kind of infections. Such individuals become more prone for tuberculous infections or reactivation of latent infection; generally affecting lungs, however disease can be disseminated in one-third of cases [2]. False-negative tuberculin tests are seen in immunosuppressed individuals hence the transplant patients as well as patients on dialysis who have or had tuberculosis are at increased risk of being not diagnosed $[2,40]$ causing further delay in treatment.

\section{Complications of renal TB}

\section{Extra Renal Spread}

The TB disease process may spread to the perinephric and retroperitoneal areas. CT adequately images the spread to perinephric, paranephric spaces $[12,14,41]$. Spread to perinephric space and retroperitoneal areas like psoas muscle involvement [41] is commonly seen with 

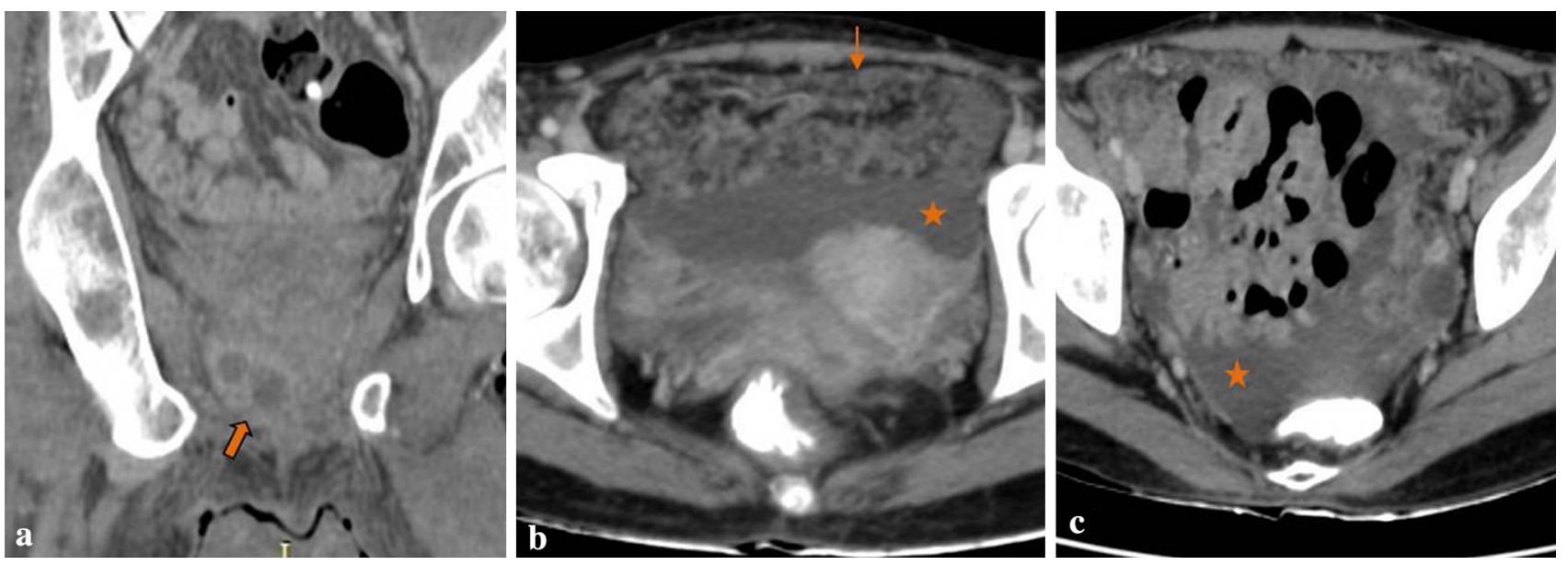

Fig. 13 Post contrast coronal (a) and axial (b) and CT (c). Prostatic abscess in (thick arrow), omental thickening-omental cake formation (thin arrow) and ascites (star)

Fig. 14 Post-contrast axial (a) and Sagittal MPR (b). CT reveals aleft renal abscess that has ruptured in perirenal space (thin arrow) and into the psoas abscess (thick arrow). Note in b the psoas abscess has superiorly displaced the kidney
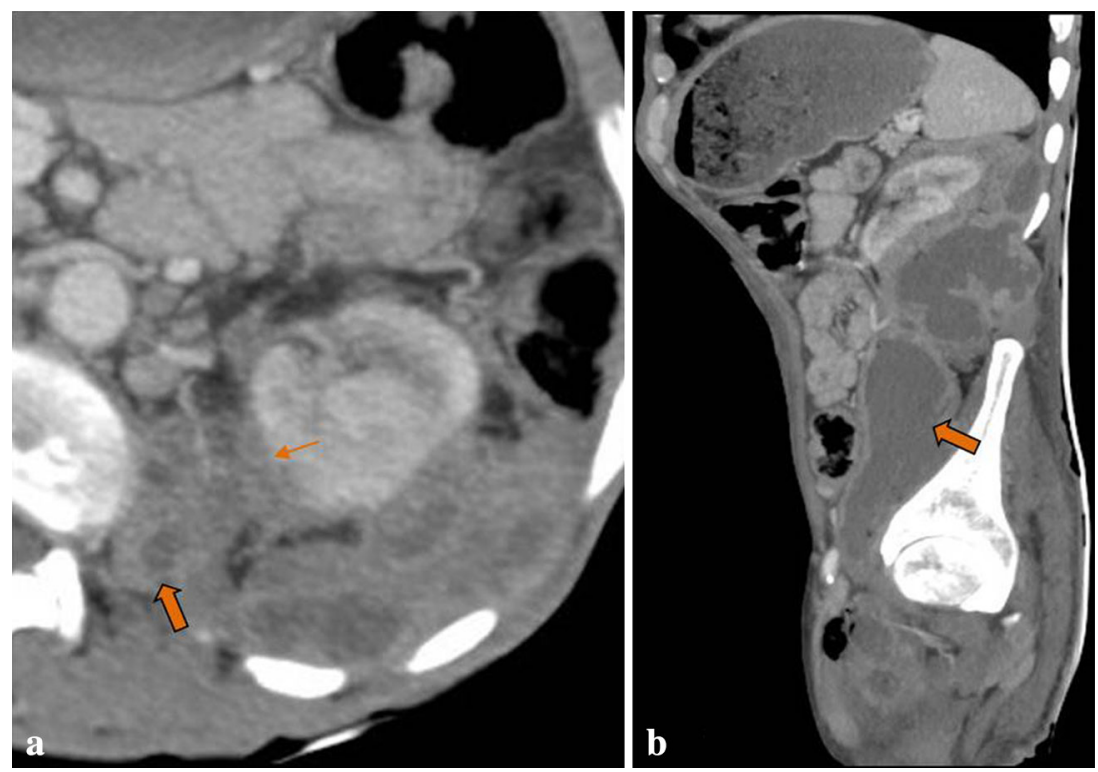

disseminated TB. Figure 14 reveals psoas muscle and perinephric abscesses formation. Adrenal involvement; retroperitoneal collections; subcutaneous collections; retroperitoneal fibrosis; and prostate, seminal vesicle, and spinal affection are all extremely well evaluated by CT [41, 42].

Fistulas may be seen which extend even beyond these retroperitoneal confines, including into the gastrointestinal tract, skin, lymphatic vessels, and thoracic cavity (pleura, bronchus) [43]. Renal TB causing a liver abscess has been reported [44].

\section{Amyloidosis}

Secondary amyloidosis is related to chronic infection or inflammation. Chronic TB is an important and commonest cause in India which can rarely be complicated by amyloidosis causing renal disease [45]. Secondary amyloidosis results in renal failure. Prompt treatment of the underlying TB focus can prevent progression to end-stage renal disease. There is decrease in the recent incidence of renal amyloidosis due to TB because of availability of effective antituberculous drugs.

Squamous Metaplasia

Chronic inflammation and infection of the renal pelvis can lead to Keratinizing squamous metaplasia as a complication and may persist even after treatment of the active tuberculous lesion [2, 46]. This is a potential risk factor for the development of squamous carcinoma in chronic cases. 


\section{End-Stage Renal Disease}

End-stage renal disease in renal TB can occur due to obstructive uropathy as a result of multiple strictures especially at pelviureteric junction. Secondary amyloidosis or obliterative endarteritis of intrarenal vessels is the other cause [47]. Disseminated Tuberculosis seen commonly with immunocompromised individuals may affect the kidney resulting in granulomatous interstitial nephritis eventually leading to renal failure [2].

Certain biochemical abnormalities are seen with renal TB in end-stage renal disease; hypercalcemia being one of them. Hypercalcemia is secondary to abnormal/elevated calcitriol production by the granulomatous tissue [2, 47].

Tuberculosis is an uncommon cause of renal failure which is treatable and potentially preventable. The overall incidence of renal failure related to $\mathrm{TB}$, reported in the literature is $24 \%$ [47].

Acute Interstitial Nephritis as the term suggest involves renal tubules and/or interstitium sparing the glomerulus. It is characterized histopathologically by inflammation and edema of the renal interstitium [48]. Acute interstitial nephritis (AIN) is due to cellular injury caused by viral, fungal or bacterial infections or as a hypersensitivity reaction to drugs or by immunologic conditions. AIN rapidly and abruptly causes the renal failure [47, 48].

Granulomatous interstitial nephritis $(G I N)$ is an uncommon form of acute interstitial nephritis [48]; tuberculosis being the rare cause. Pathology is granuloma formation with epithelioid giant cells usually found in AIN secondary to tuberculosis, sarcoidosis or Wegener's granulomatosis [48]. AIN can also be caused due to RIFAMPICIN drug used in treatment of TB [47]. Renal biopsy is the definitive test for diagnosis of AIN [48, 49].

As the tubules have regenerative capacity, the renal function in AIN can be made reversible/or maintained if the diagnosis is made before complete deterioration of renal function occurs using a combination of anti-tubercular treatment and corticosteroids [2].

\section{Conclusion}

Renal tuberculosis is one of the commonest extrapulmonary form, frequently seen in endemic areas and is not easily diagnosed clinically due to absence of classical symptoms. It is also one of the treatable cause of renal failure. Hence the radiologist and imaging assumes an important role in reaching the diagnosis.

Computed tomography is highly sensitive for detection of calcification which is commonly seen with TB. With advent of newer generation Multidetector scanners, increased resolution has resulted in increased sensitivity and detection of renal lesions. CT urography performed on the current highend MDCT scanners have the potential to detect early TB changes like papillary necrosis that were usually noted on IVU. Though individual features may not help in diagnosis; multiple cortical lesions, parenchymal scarring and pelvicalyceal system involvement in form of papillary necrosis, lobar caseation, calcification, uneven caliectasis and strictures are pathogonomic of renal TB. Awareness of these imaging findings assists in making the diagnosis which is further confirmed by histopathology. Thus CT imaging will continue to play an important role in establishing the initial diagnosis as well as in follow up studies.

Acknowledgements We acknowledge Dr Ashank Bansal for preparing the images and Prof Suleman Merchant, HOD dept of Radiology, LTMGH and LTMMC, Sion, Mumbai, India.

\section{Compliance with Ethics Guidelines}

Conflict of Interest Dr. Ashwini Sankhe declares no potential conflicts of interest. Dr. Anagha R. Joshi is a section editor for Current Radiology Reports.

Human and Animal Rights and Informed Consent This article does not contain any studies with human or animal subjects performed by any of the authors.

\section{References}

Papers of particular interest, published recently, have been highlighted as:

- Of importance

- Of major importance

1. Das P, Ahuja A, Gupta SD. Incidence, etiopathogenesis and pathological aspects of genitourinary tuberculosis in India: A journey revisited. Indian J Urol. 2008;24(3):356-61.

2. Eastwood JB, Corbishley CM, Grange JM. Tuberculosis and the kidney. JASN. 2001;12(6):1307-14.

3. Chauhan LS, Tonsing J. Revised national TB control programme in India. Tuberculosis (Edinb). 2005;85:271-6.

4. Chijioke A. Current concepts on pathogenesis of renal tuberculosis. West Afr J Med. 2001;20(2):107-10.

5. Langemeier Jane. Tuberculosis of the genitourinary system. Urol Nurs. 2007;27(4):279-84.

6. Pasternak MS, Rubin RH. Urinary tract tuberculosis. In: Schrier RW, editor. Diseases of the kidney and urinary tract. 7th ed. Philadelphia: Lippincott Williams \& Wilkins; 2001. p. 1017-37.

7. Sechi LA, Pinna MP, Sanna A, Pirina P, Ginesu F, Saba F, Aceti A, Turrini F, Zanetti S, Fadda G. Detection of Mycobacterium tuberculosis by PCR analysis of urine and other clinical samples from AIDS and non-HIV-infected patients. Mol Cell Probes. 1997;11:281-5.

8. Aceti A, Zanetti S, Mura MS, Sechi LA, Turrini F, Saba F, Babudieri S, Mannu F, Fadda G. Identification of HIV patients with active pulmonary tuberculosis using urine based polymerase chain reaction assay. Thorax. 1999;54:145-6.

9. Gibson MS, Puckett ML, Shelly ME. Renal tuberculosis. Radiographics. 2004;24:251-6. 
10. - Merchant S, Bharati A, Merchant N. Tuberculosis of the genitourinary system-Urinary tract tuberculosis: renal tuberculosisPart II. Genitourin Obstet Radiol. 2013; 23(1):64-7. The reference is latest and gives comprehensive information about genitourinary Koch's.

11. Quaia E. Imaging findings in renal tuberculosis on computed tomography urography. Eur Urol Rev. 2010;5(2):69-72. This reference article is one of the latest article about CT in Renal Koch's and included as it is within 5 years.

12. Wang LI, Wong YC, Chen CJ, Lim KE. CT features of genitourinary tuberculosis. J Comput Assist Tomogr. 1997;21:254-8.

13. Hartman DS, Stagg PL. Diagnosis please. Case 3: renal tuberculosis. Radiology. 1998;209:69-72.

14. Goldman SM, Fishman EK, Hartman DS, Siegelman SS. Computed tomography of renal tuberculosis and its pathological correlates. J Comput Assist Tomogr. 1985;9:771-6.

15. Kim SH. Urogenital tuberculosis. In: Pollack HM, McClennan BL, editors. Clinical urography. 2nd ed. Philadelphia: WB Saunders Co; 2000. p. 1193-228.

16. Wang LJ, Wu CF, Wong YC, Chuang CK, Chu SH, Chen CJ. Imaging findings of urinary tuberculosis on excretory urography and computerized tomography. J Urol. 2003;169:524-8.

17. Goldman SM, Fishman EK. Upper urinary tract infection: the current role of CT, ultrasound, and MRI. Semin Ultrasound CT MR. 1991;12:335-60.

18. Engin G, Acuna B, Acuna G, Tunaci M. Imaging of extrapulmonary tuberculosis. Radiographics. 2000;20:471-88.

19. Li Y, Yang ZG, Guo YK, Min PQ, Yu JQ, Ma ES, et al. Distribution and characteristics of hematogenous disseminated tuberculosis within the abdomen on contrast-enhanced CT. Abdom Imaging. 2007;32:484-8.

20. Harisinghani MG, McLoud TC, Shepard JA, Ko JP, Shroff MM, Mueller PR. Tuberculosis from head to toe. Radiographics. 2000;20:449-70.

21. Buxi TB, Sud S, Vohra R. CT and MRI in the diagnosis of tuberculosis. Indian J Pediatr. 2002;69:965-72.

22. Lu P, Li C, Zhou X. Significance of the CT scan in renal tuberculosis. Zhonghua Jie He He Hu Xi Za Zhi. 2001;24:407-9.

23. Kawashima A, Sandler CM, Ernst RD, Goldman SM, Raval B, Fishman EK. Renal inflammatory disease: the current role of CT. Crit Rev Diagn Imaging. 1997;38:369-415.

24. Miller FH, Parikh S, Gore RM, Nemcek AA Jr, Fitzgerald SW, Vogelzang RL. Renal manifestations of AIDS. Radiographics. 1993;13:587-96.

25. Lang EK, Macchia RJ, Thomas R, Watson RA, Marberger M, Lechner $\mathrm{G}$, et al. Improved detection of renal pathologic features on multiphasic helical CT compared with IVU in patients presenting with microscopic hematuria. Urology. 2003;61:528-32.

26. Jung DC, Kim SH, Jung SI, Hwang SI, Kim SH. Renal papillary necrosis: review and comparison of findings at multi-detector row CT and intravenous urography. Radiographics. 2006;26:1827-36.

27. Lang EK, Macchia RJ, Thomas R, et al. Detection of medullary and papillary necrosis at an early stage by multiphasic helical computerized tomography. J Urol. 2003;170(1):94-8.

28. Bittar EE, Misanik L. Renal necrotizing papillitis. Am J Med. 1963;34:82-7.

29. Kenney PJ. Imaging of chronic renal infections. AJR Am J Roentgenol. 1990;155:485-94.
30. Okazawa N, Sekiya T, Tada S. Computed tomographic features of renal tuberculosis. Radiat Med. 1985;3:209-13.

31. Zissin R, Gayer G, Chowers M, Shapiro-Feinberg M, Kots E, Hertz M. Computerized tomography findings of abdominal tuberculosis: report of 19 cases. Isr Med Assoc J. 2001;3:414-8.

32. Figueiredo AA, Lucon AM, Júnior RF, Ikejiri DS, Nahas WC, Srougi M. Urogenital tuberculosis in immunocompromised patients. Int Urol Nephrol. 2009;41:327-33.

33. Eastwood JB, Corbishley CM, Grange JM. Tuberculosis and the kidney. J Am Soc Nephrol. 2001;12:1307-14.

34. Lanjewar DN, Ansari MA, Shetty CR, Maheshwary MB, Jain P. Renal lesions associated with AIDS-An autopsy study. Indian J Pathol Microbiol. 1999;42:63-8.

35. Soriano-Rosas J, Avila-Casado MC, Carrera-Gonzalez E, Chavez-Mercado L, Cruz-Ortiz H, Rojo J. AIDS-associated nephropathy: 5-year retrospective morphologic analysis of 87 cases. Pathol Res Pract. 1998;194:567-70.

36. Wise GJ. Urinary tuberculosis: modern issues. Curr Urol Rep. 2009;10:313-8.

37. Davies PDO. Vitamin D and tuberculosis. Am Rev Respir Dis. 1989;139:1571.

38. Wilkinson RJ, Llewelym M, Toossi Z, Patel P, Pasvol G, Lalvani A, Wright D, Latif M, Davidson RN. Influence of vitamin D deficiency and vitamin D receptor polymorphisms on tuberculosis among Gujarati Asians in west London: a case-control study. Lancet. 2000;355:618-21.

39. Singh N, Paterson DL. Mycobacterium tuberculosis infection in solid-organ transplant recipients: impact and implications for management. Clin Infect Dis. 1998;27:1266-77.

40. Woeltje KF, Mathew A, Rothstein M, Seiler S, Fraser VJ. Tuberculosis infection and anergy in hemodialysis patients. Am J Kidney Dis. 1998;31:848-52.

41. Merchant SA. Tuberculosis of the genitourinary system. Indian J Radiol Imaging. 1993;3:253-74.

42. Benchekroun A, Lachkar A, Soumana A, Farih MH, Belahnech Z, Marzouk M, et al. Urogenital tuberculosis. 80 cases. Ann Urol (Paris) 1998;32:89-94.

43. Jafri SZ, Roberts JL, Berger BD. Fistulas of the genitourinary tract. In: Pollack HM (ed) Clinical urography. 2nd edn. Saunders, Philadelphia. 2000. pp. 2992-3011.

44. Shah HN, Jain P, Chibber PJ. Renal tuberculosis simulating xanthogranulomatous pyelonephritis with contagious hepatic involvement. Int J Urol. 2006;13:67-8.

45. Chugh KS, Datta BN, Singhal PC, Jain SK, Sakhuja V, Dash SC. Pattern of renal amyloidosis in Indian patients. Postgrad Med J. 1981;57:31-5.

46. Byrd RB, Viner NA, Omell GH, Trunk G. Leukoplakia associated with renal tuberculosis in the chemotherapeutic era. $\mathrm{Br} \mathrm{J}$ Urol. 1976;48:377-81.

47. Krishnamoorthy S, Gopalakrishnan G. Surg Manag Renal Tuberc. Indian J Urol Symp 2008;24(3):369-75.

48. Kodner CM, Kudrimoti A. University of Louisville School of Medicine, Louisville, Kentucky. Diagnosis and Management of Acute Interstitial Nephritis. Am Fam Physician. 2003;67(12):2527-34.

49. Sampathkumar K, Sooraj YS, Mahaldar AR, Ramakrishnan M, Rajappannair A et al. Granulomatous interstitial nephritis due to tuberculosis-a rare presentation. Saudi J Kidney Dis Transplant. 2009;20(5):842-5. 